\title{
Axon Branching Requires Interactions between Dynamic Microtubules and Actin Filaments
}

\author{
Erik W. Dent ${ }^{1}$ and Katherine Kalii ${ }^{1,2}$ \\ ${ }^{1}$ Neuroscience Training Program and ${ }^{2}$ Department of Anatomy, University of Wisconsin, Madison, Wisconsin 53706
}

Cortical neurons innervate many of their targets by collateral axon branching, which requires local reorganization of the cytoskeleton. We coinjected cortical neurons with fluorescently labeled tubulin and phalloidin and used fluorescence timelapse imaging to analyze interactions between microtubules and actin filaments (F-actin) in cortical growth cones and axons undergoing branching. In growth cones and at axon branch points, splaying of looped or bundled microtubules is accompanied by focal accumulation of F-actin. Dynamic microtubules colocalize with F-actin in transition regions of growth cones and at axon branch points. In contrast, F-actin is excluded from the central region of the growth cone and the axon shaft, which contains stable microtubules. Interactions between dynamic

Axons are guided in new directions by reorientation of their growth cones as well as extension of collateral branches (O'Leary et al., 1990). We have shown previously (Szebenyi et al., 1998) that cortical axon branching occurs in vitro through changes in growth cone morphologies and behaviors. Growth cones at the tips of rapidly extending cortical axons are typically small and highly motile. However, in preparation for branching, growth cones pause for many hours, greatly enlarge, and maintain motility without forward advance. Subsequently, a new growth cone develops from the tip of the large paused growth cone and forms the new leading axon. Remnants of the large paused growth cone remain behind on the axon shaft as filopodial and lamellar expansions that subsequently give rise to interstitial axon collaterals. In living cortical slices (Halloran and Kalil, 1994), similar growth cone pausing behaviors were observed in the corpus callosum in regions where collateral axon branches develop and extend toward cortical targets, suggesting that growth cone pausing is closely related to branching mechanisms in vivo.

Changes in the direction of axon outgrowth depend on reorganization of the microtubule and actin cytoskeleton (Lin and Forscher, 1993; Tanaka et al., 1995; Challacombe et al., 1996, 1997; Williamson et al., 1996; Suter et al., 1998; Gallo and Letourneau, 1999). Microtubules in the central region of advancing growth cones are splayed apart but become bundled and form loops in slowly growing axons (Tsui et al., 1984; Sabry et al., 1991;

Received July 6, 2001; revised Aug. 29, 2001; accepted Sept. 26, 2001.

This work was funded by National Institutes of Health Grants NS14428 and NS34270 and Predoctoral Training Grant GM07507 to E.W.D. We thank Wenqian $\mathrm{Yu}$ for performing the electron microscopy for Figure 2. We also thank William Bement, Timothy Gomez, Helen Lueth, and Gerard Marriott for comments on this manuscript.

Movies of several figures can be viewed at http://kalil.anatomy.wisc.edu.

Correspondence should be addressed to Dr. Katherine Kalil, University of Wisconsin, Department of Anatomy, 1300 University Avenue, Madison, WI 53706. E-mail: kakalil@facstaff.wisc.edu.

Copyright (C) 2001 Society for Neuroscience $0270-6474 / 01 / 219757-13 \$ 15.00 / 0$ microtubules and dynamic actin filaments involve their coordinated polymerization and depolymerization. Application of drugs that attenuate either microtubule or F-actin dynamics also inhibits polymerization of the other cytoskeletal element. Importantly, inhibition of microtubule or F-actin dynamics prevents axon branching but not axon elongation. However, these treatments do cause undirected axon outgrowth. These results suggest that interactions between dynamic microtubules and actin filaments are required for axon branching and directed axon outgrowth.

Key words: microtubule; actin filament; growth cone; collateral axon branching; cortical development; fluorescence timelapse imaging

Tanaka and Kirschner, 1991). In a previous study using live cell imaging, we found that microtubules form prominent loops in the central region of large paused growth cones. Transition to new axonal growth and branch formation is accompanied by splaying of looped microtubules and formation of short microtubule fragments that invade the lamellipodium (Dent et al., 1999). Similar reorganization of the microtubule array also occurs at developing branch points along the axon shaft (Kalil et al., 2000). Although actin is known to play an important role in regulating the distribution of microtubules, the exact nature of F-actin-microtubule interactions in the growth cone is not well understood (Suter and Forscher, 2000) and has not been well characterized in motile cells (Waterman-Storer and Salmon, 1999).

Much of our understanding of how actin filaments and microtubules reorganize during directed axon outgrowth is based on interpretations from fixed preparations. Some studies have used live cell imaging to characterize dynamic changes in the microtubule array or in F-actin, but the approach has been to visualize one or the other cytoskeletal element in living neurons and then to determine accompanying changes in the other element by rapid fixation and staining (Challacombe et al., 1997; Suter et al., 1998; Rochlin et al., 1999; Kabir et al., 2001). However, associations between $\mathrm{F}$-actin and microtubules are likely to be highly dynamic. Therefore, in the present study, we have used the novel approach of visualizing simultaneous changes in both microtubules and actin filaments in relation to one another during different stages of axon branching in living cortical neurons.

\section{MATERIALS AND METHODS}

Cell culture. Cultures were prepared from cortical tissue obtained from the brains of 2- to 3-d-old golden Syrian hamsters (Mesocricetus auratus) as described previously (Dent et al., 1999). For some experiments low oxygen conditions $\left(37^{\circ} \mathrm{C}, 5 \% \mathrm{CO}_{2}\right.$ and $\left.9 \% \mathrm{O}_{2}\right)$ were used to more closely approximate in vivo conditions (Lubbers et al., 1994).

Injection of fluorescent probes. Tubulin was prepared from bovine brain 


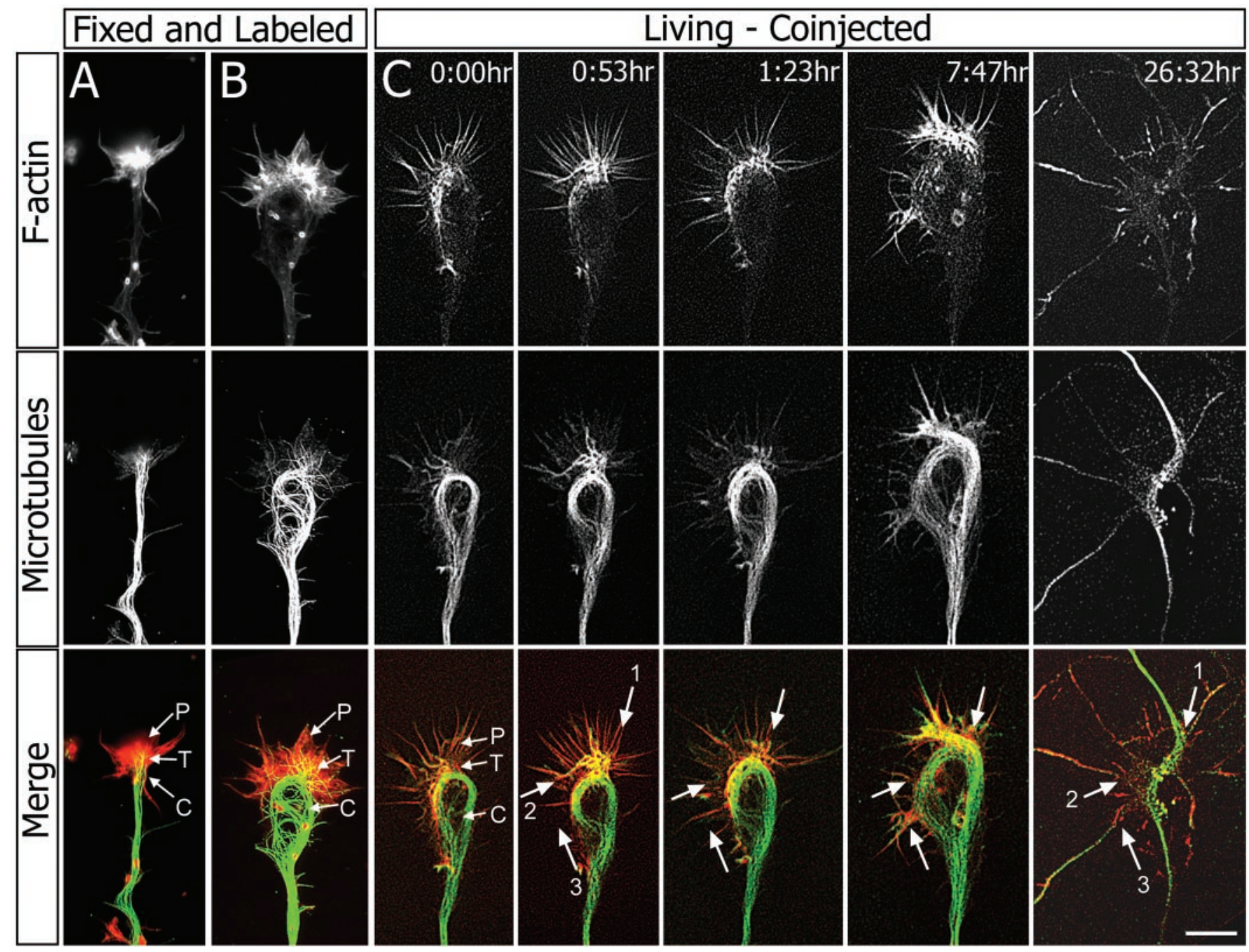

Figure 1. Colocalization of microtubules and F-actin in regions of branching from the growth cone. $A$, Fixed cortical neuron labeled for both F-actin (phalloidin) and microtubules (antityrosinated $\alpha$-tubulin). F-actin is present throughout the peripheral $(P)$, transition $(T)$, and central $(C)$ regions of the growth cone. Microtubules are concentrated in the axon shaft and central region and splay out into the peripheral region. In merged images in $A-C$, F-actin is shown in red, microtubules in green, and their overlap in yellow. B, Fixed cortical neuron with a large paused growth cone labeled for F-actin (phalloidin) and microtubules (antityrosinated $\alpha$-tubulin). Microtubules form a prominent loop in the central region of the growth cone, and F-actin is present in the transition and peripheral regions but excluded from the central regions of the growth cone. $C$, Series of time-lapse images of a living neuron coinjected with fluorescent phalloidin and tubulin. The growth cone is paused and shows distribution of F-actin and microtubules similar to that in the fixed paused growth cone in B. Arrows $(0: 53 \mathrm{hr}-26: 32 \mathrm{hr})$ show positions of the new axon (arrow 1) and two prominent branches (arrows 2, 3) that develop from the growth cone. Scale bar, $10 \mu \mathrm{m}$.

(Hyman et al., 1991) and labeled with tetramethyl-rhodamine (TMR) as described previously (Keating et al., 1997). In some experiments, rhodamine-tubulin was purchased (Cytoskeleton Inc.). Actin filaments in cortical neurons were labeled by injection of Alexa 488-phalloidin (Molecular Probes, Eugene, OR). Alexa 488-phalloidin was stored at $-20^{\circ} \mathrm{C}$ as a $6.6 \mu \mathrm{M}$ methanol stock. For injections, $200 \mu \mathrm{l}$ of phalloidin was dried under nitrogen and resuspended in $0.5 \mu \mathrm{l}$ of dry DMSO. TMR-tubulin was diluted to $5-10 \mathrm{mg} / \mathrm{ml}$ in injection buffer (100 mM PIPES and $0.5 \mathrm{~mm}$ $\mathrm{MgCl}_{2}, \mathrm{pH}$ 6.9), and $9.5 \mu \mathrm{l}$ was added to the phalloidin-DMSO mixture, resulting in final concentrations of $132 \mu \mathrm{M}$ Alexa 488-phalloidin and $4.8-9.5 \mathrm{mg} / \mathrm{ml}$ TMR-tubulin in the injection pipette (Sanders and Wang, 1991). Injection of the phalloidin-tubulin mixture and preparation of injected neurons for live cell imaging were performed as described previously (Dent et al., 1999).

Time-lapse fluorescence imaging. Long-term $(>1 \mathrm{hr})$ live cell imaging was performed by projecting images of fluorescently labeled cells through a Keller port of a Zeiss (Thornwood, NY) Axiovert 135M inverted microscope equipped with a Photometrics PXL slow-scan liquid cooled CCD camera containing an Eastman Kodak Co. KAF-1400 chip (Roper Scientific). To image both the Alexa 488-phalloidin-labeled F-actin and the TMR-labeled microtubules a Lambda 10-2 dual filter wheel (Sutter Instruments, Novato, CA) was attached to both the excitation and emission ports of the microscope, and a multiple bandpass dichroic filter was installed in the Zeiss 3FL slider. These filter wheels were equipped with Chroma 61005 excitation-emission filter sets capable of exciting FITC, $\mathrm{Cy} 3$, and $\mathrm{Cy} 5$ wavelength dyes in quick succession and almost perfect register with little overlap between channels (Salmon et al., 1998). To determine the amount of cross-talk between channels, either $132 \mu \mathrm{M}$ Alexa 488-phalloidin or $10 \mathrm{mg} / \mathrm{ml}$ TMR-tubulin was injected into single cells. Images were collected in both channels, and pixel intensities were measured in areas of growth cones and axons. For neurons injected with only one label, the pixel intensities in the unlabeled channel were always between 0.1 and $1.8 \%$ above background. This represents negligible cross-talk between filters. Illumination during epifluorescence imaging was attenuated to $10-20 \%$ of the output of the $100 \mathrm{~W}$ mercury lamp by means of neutral density filters (Chroma). Electronically controlled shutters (Uniblitz; Vincent Associates) limited the illumination to the period of image acquisition. Well labeled, motile neurons were imaged with a $100 \times 1.3$ numerical aperture (NA) Plan Fluor objective (Zeiss). Images were acquired every $30 \mathrm{~min}$ to $4 \mathrm{hr}$ with $250-1000 \mathrm{msec}$ exposures. The 
camera, shutters, filter wheels, and focus motor were all controlled by Metamorph Software (Universal Imaging Corp., West Chester, PA). All images were saved in 12 bit format.

For short-term imaging $(<1 \mathrm{hr})$, a separate microscope and camera system was used to image fluorescent microtubules and F-actin in register and in quick succession (1-2 sec between exposures) in the same neuron. Images of labeled neurons were projected through a Keller port of a Nikon TE300 Quantum inverted microscope equipped with a Princeton Instruments MicroMax 512BFT cooled CCD camera containing a backthinned, frame transfer EEV CCD57-10 chip (Roper Scientific). This camera allowed for rapid acquisition (100-500 msec) of very low light level images (5-10\% mercury light output) but maintained sufficient resolution (13.0 $\mu \mathrm{m}^{2}$ pixels). Filter wheels and shutters were attached to the microscope in the same configuration as above. Neurons were imaged in time lapse (5-15 sec intervals) with a $100 \times 1.4$ NA Plan Apo CFI60 objective (Nikon). This objective is chromatically corrected so that the Alexa 488-phalloidin-labeled actin filaments and TMR-labeled microtubules could be imaged without adjusting focus. All peripherals were controlled by Metamorph. Images were collected and saved in 16 bit format.

Long-term drug treatments and immunocytochemistry. For all long-term treatments, drugs were added to cortical cultures $18 \mathrm{hr}$ after plating to minimize any possible effects on the establishment of polarity. Neurons were exposed to the following drugs for $30 \mathrm{hr}$ : $10 \mathrm{~nm}$ taxol (Sigma, St. Louis, MO), $33 \mathrm{~nm}$ nocodazole (Aldrich, Milwaukee, WI), $0.5 \mu \mathrm{M}$ latrunculin A (Molecular Probes), and $1 \mu \mathrm{M}$ cytochalasin B (Sigma). Some cultures were fixed (48 hr in culture) for $15 \mathrm{~min}$ in $4 \%$ paraformaldehyde (EM Sciences) in Krebs' buffer with 0.4 M sucrose to preserve both F-actin and cytoplasmic globular actin (G-actin) (Dent and Meiri, 1992). These neurons were stained for both F- and G-actin by simultaneously incubating the cultures with $0.33 \mu \mathrm{M}$ Alexa 488-phalloidin (Molecular Probes) and $20 \mu \mathrm{M}$ rhodamine-DNase 1 (Molecular Probes) for 1 hr. Cultures were then mounted in $80 \%$ glycerol and PBS. To quantify the distributions of tyrosinated and acetylated microtubules in relation to F-actin, cortical neuronal cultures were simultaneously extracted and fixed to preserve the majority of F-actin and microtubules but to extract cytoplasmic G-actin and tubulin (Challacombe et al., 1996; Williamson et al., 1996). This microtubule-F-actin fixative was composed of $4 \%$ paraformaldehyde, $0.25 \%$ glutaraldehyde (EM Sciences), $0.1 \%$ Triton X-100 (Sigma), $10 \mu \mathrm{M}$ taxol (Sigma), and $1.3 \mu \mathrm{M}$ phalloidin (Molecular Probes) in PHEM buffer (60 mM PIPES, $25 \mathrm{~mm}$ HEPES, $10 \mathrm{~mm}$ EGTA, and 2 $\mathrm{mM} \mathrm{MgCl}_{2}, \mathrm{pH}$ 6.9). Cortical cultures were simultaneously labeled with a rat monoclonal antibody to tyrosinated $\alpha$-tubulin (YL 1/2; Chemicon, Temecula, CA) at 1:1000 and a mouse monoclonal antibody to acetylated $\alpha$-tubulin (6-11B-1; Sigma) at 1:1000. Cultures were then simultaneously incubated with $\mathrm{Cy} 2$ donkey anti-rat and $\mathrm{Cy} 5$ donkey anti-mouse secondary antibodies, both at 1:200 (Jackson ImmunoResearch, West Grove, PA). Cells were subsequently labeled with $0.33 \mu \mathrm{M}$ rhodamine-phalloidin (Molecular Probes) and mounted in $80 \%$ glycerol and PBS.

Image processing and data analysis. For quantification of branch length, branch number, and axon length, images of fixed cells were acquired with a $20 \times 0.5$ NA (CFI60) Plan Fluor objective. Axon lengths were measured from the cell body to the distal extent of the central region of the growth cone. An axon was defined as a process that remained parallel to the initial axon segment extending from the cell body. Branches were defined as processes extending at orthogonal angles to the axon.

To measure levels of staining for microtubules and F-actin semiquantitatively, 16 bit images of fluorescently labeled growth cones and branches were flat field-corrected by means of the correct shading function in Metamorph. The amount of colocalization between microtubules and F-actin was determined with the colocalization function in Metamorph. F-actin-to-G-actin ratios were computed by dividing images of F-actin by images of G-actin. All multicolor images were merged with the overlay images function in Metamorph.

To determine the dynamic relationship between F-actin and microtubules in living growth cones and branches, movies of montaged images were constructed and analyzed frame by frame. The tips of both microtubules and actin filaments were tracked using the track points function in Metamorph. The change in position of microtubule and F-actin tips from frame to frame was calculated with the equation $\left(x_{1}-x_{2}\right)^{2}+\left(y_{1}-\right.$ $\left.y_{2}\right)^{2}=$ distance moved, where $\left(x_{1}, y_{1}\right)$ is the position of the microtubule or F-actin tip in a frame, and $\left(x_{2}, y_{2}\right)$ is the position of the microtubule or F-actin tip in the next frame. Tips of actin filaments and microtubules were considered coextensive if they were within $0.9 \mu \mathrm{m}$ of each other. This value was computed using $0.3 \mu \mathrm{m}$ as the resolvable distance be-
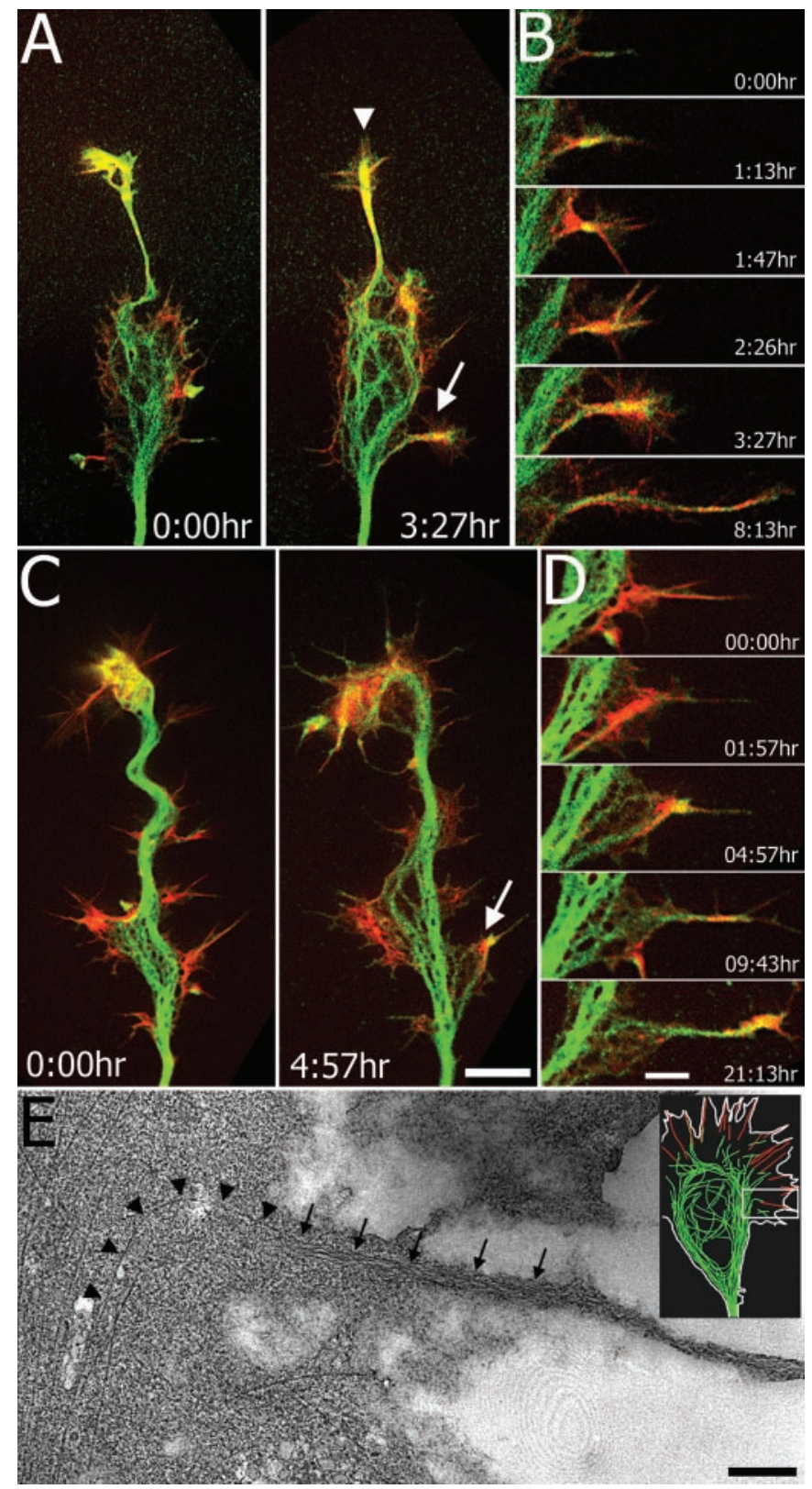

Figure 2. Reorganization of microtubules and F-actin during development of branches from the growth cone and axon shaft. $A$, Lower-power time-lapse images of the same neuron at two time points showing a new axon, not present when the neuron was injected, forming from the distal tip of the large paused growth cone. The arrowhead indicates the growth cone at the tip of the developing axon (3:27hr). In $A-D$, F-actin is shown in red, microtubules in green, and their overlap in yellow. $B$, Series of higher-power time-lapse images of the developing branch $(A$, arrow at 3:27hr) on the growth cone shown in $A$. The branch begins as a filopodium containing few microtubules and F-actin $(0: 00 \mathrm{hr})$. Later $(1: 13 \mathrm{hr}-3: 27 \mathrm{hr})$, microtubules and F-actin colocalize (yellow) in the growth cone at the tip of the branch. $C$, Lower-power time-lapse images showing development of branches from the axon shaft. At each branch point, F-actin is concentrated in regions where microtubules splay apart. $D$, Series of higherpower images of the developing branch (arrow at 4:57hr) on the axon shown in $C$. Microtubules splay apart and invade the developing branch $(4: 57 h r)$. At 21:13hr, a growth cone has formed on the tip of the branch. $E$, Electron micrograph from the boxed region of a growth cone drawn in the inset demonstrating that a microtubule (arrowheads) splays from the loop and is closely apposed to a bundle of actin filaments (arrows) in the lamellipodium. Scale bars: $A, C, 10 \mu \mathrm{m} ; B, D, 5 \mu \mathrm{m} ; E, 0.5 \mu \mathrm{m}$. 

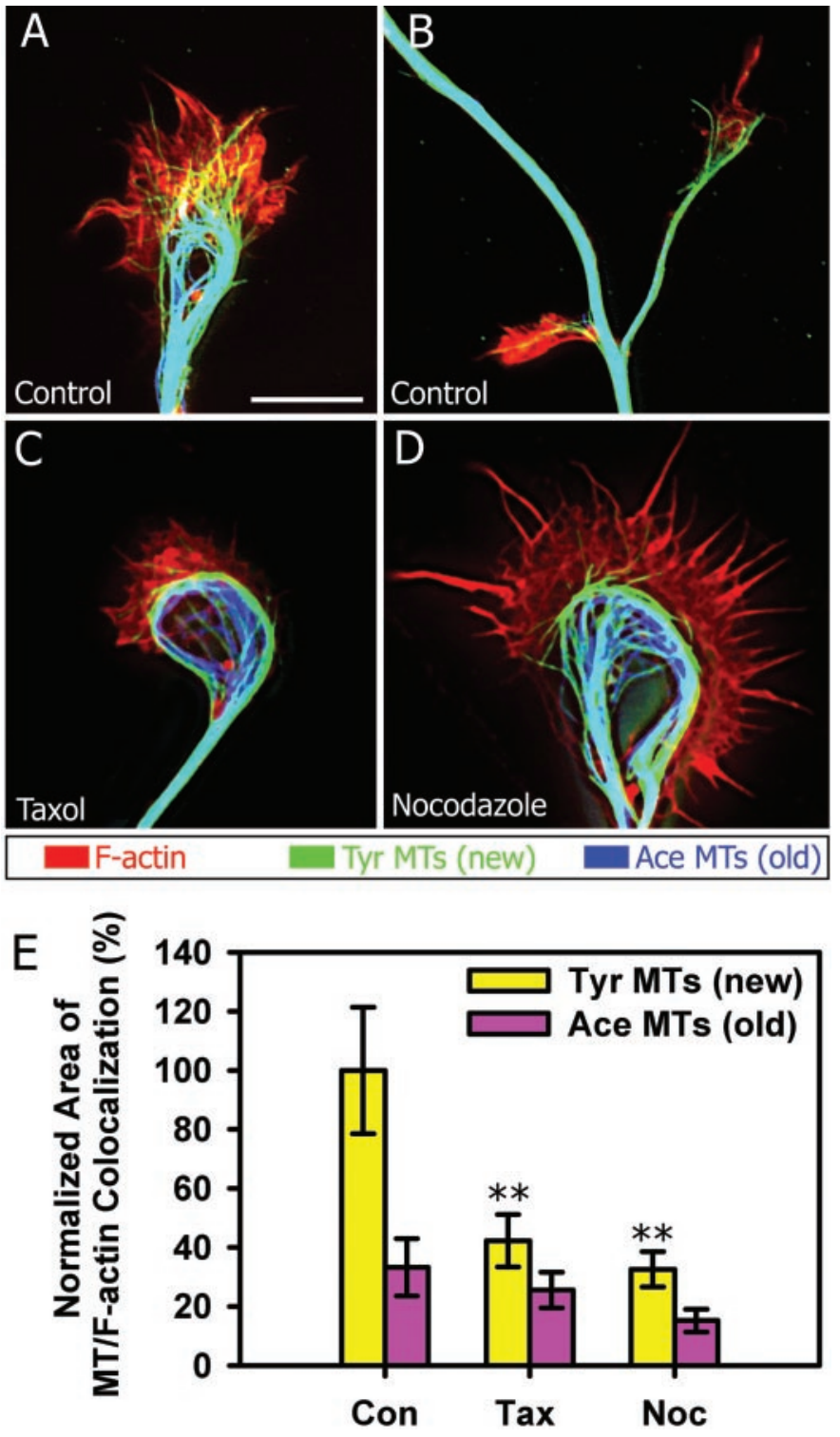

Figure 3. Localization of microtubules and F-actin in growth cones after drug treatments that attenuate microtubule and actin dynamics. $A$, Fixed cortical growth cone labeled with phalloidin, an antibody to tyrosinated $\alpha$-tubulin and an antibody to acetylated $\alpha$-tubulin, pseudocolored as indicated. Only tyrosinated microtubules ( green) colocalize (yellow) with F-actin (red) in the transition and peripheral regions of the growth cone. Microtubules in the central loop are tyrosinated and acetylated (blue). B, Fixed cortical axon with developing branches labeled with phalloidin, an antibody to tyrosinated $\alpha$-tubulin and an antibody to acetylated $\alpha$-tubulin pseudocolored as indicated. In a newly forming branch (left) and a more extended branch (right), only tyrosinated microtubules ( green) colocalize ( yellow) with F-actin at branch points. Acetylated microtubules (blue) are concentrated in the axon shaft and the proximal region of the developing branch. $C, D$, Examples of fixed growth cones treated with $10 \mathrm{~nm}$ taxol $(C)$ or $33 \mathrm{~nm}$ nocodazole $(D)$. Very few tyrosinated microtubules are present in the growth cone transition region. $E$, Graph showing the area, normalized for growth cone size, of F-actin that colocalizes with either tyrosinated or acetylated microtubules in untreated growth cones and in those treated with $10 \mathrm{~nm}$ taxol and $33 \mathrm{~nm}$ nocodazole $(n=30$ growth cones from 3 separate experiments; mean \pm SEM). Treatments with drugs that attenuate microtubule dynamics reduce only the colocalization of tyrosinated microtubules with F-actin $(* * p<0.01$, Kruskal-Wallis ANOVA with Student-Newman-Keuls post hoc comparisons). Acetylated microtubules are unaffected. MT, Microtubule; Con, Control; Tax, taxol; Noc, nocodazole. Scale bar, $10 \mu \mathrm{m}$.

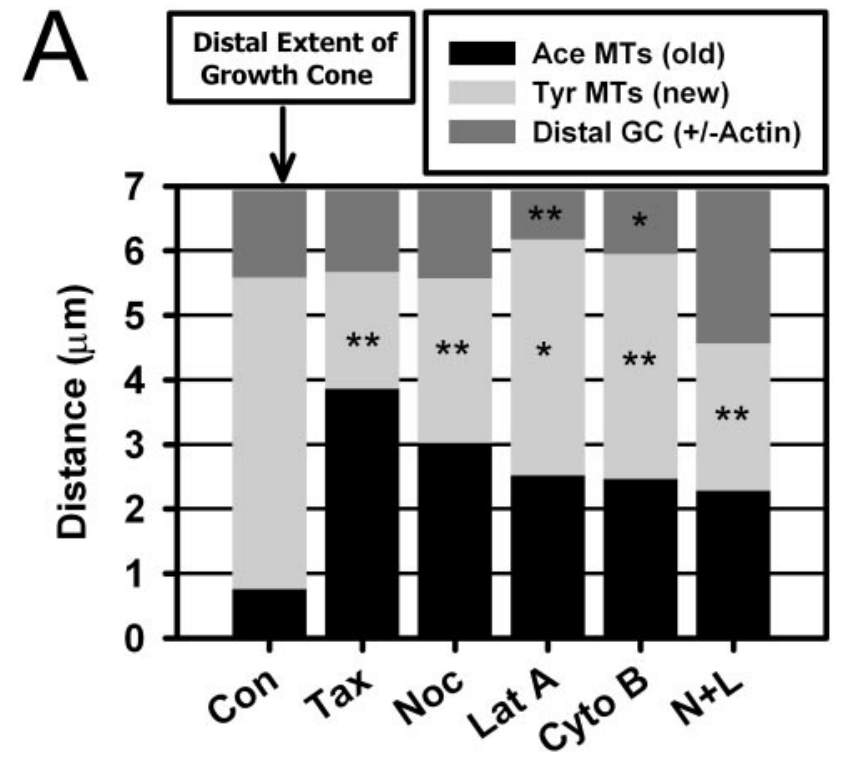

D

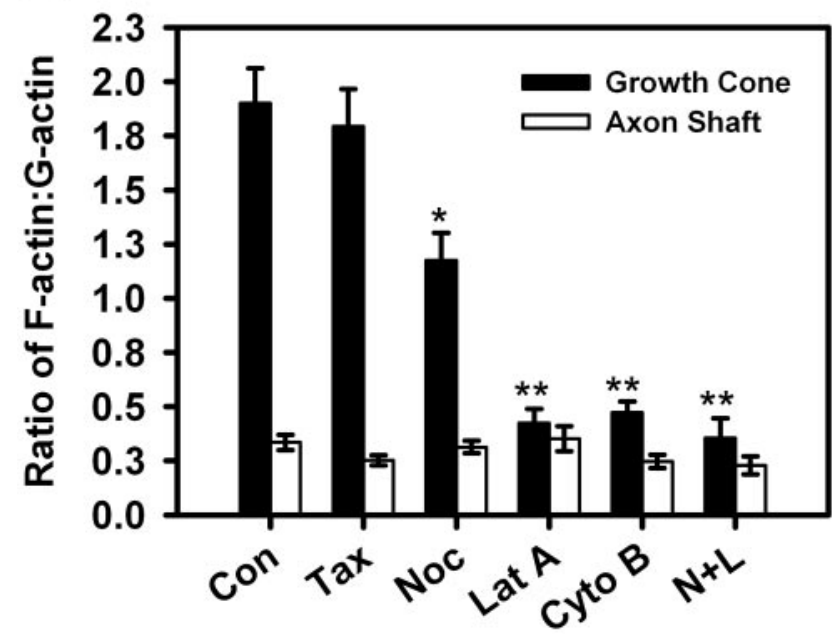

Figure 4. Drugs that decrease actin polymerization and microtubule dynamics also affect microtubules and F-actin, respectively. $A$, Graph illustrating the extent of acetylated and tyrosinated microtubules within the growth cone after treatments that attenuate microtubule dynamics or actin polymerization $(n>27$ growth cones for each treatment from 3 separate experiments). In all growth cones treated with drugs, the lengths of tyrosinated microtubule ends were significantly shorter than in controls (** $p<0.01$; Lat $A$, ${ }^{*} p<0.05$, Kruskal-Wallis one-way ANOVA on ranks with Dunn's post hoc comparisons). Treatment with actin-depolymerizing drugs also caused microtubules to extend further into the growth cone periphery in comparison with controls $\left({ }^{* *} p<0.01\right.$, Lat $A ;{ }^{*} p<0.05$, Cyto $B)$. Note that actin-depolymerizing drugs reduce the extent of actin in the distal growth cone. $B$, Bar graph illustrating the ratio of $\mathrm{F}$-actin to $\mathrm{G}$-actin in growth cones and the axon shaft $(n>30$ neurons for each treatment from 3 separate experiments). In growth cones treated with $0.5 \mu \mathrm{M}$ latrunculin $\mathrm{A}$ and $1.0 \mu \mathrm{M}$ cytochalasin $\mathrm{B}$, the ratio of $\mathrm{F}$-actin to G-actin is significantly decreased compared with controls $\left({ }^{* *} p<0.01\right.$ compared with control with Kruskal-Wallis one-way ANOVA on ranks with Dunn's post hoc comparisons). Treatment with $33 \mathrm{~nm}$ nocodazole also significantly decreased the F-actin-to-G-actin ratio in the growth cone $\left({ }^{*} p<\right.$ 0.05 compared with control with Kruskal-Wallis one-way ANOVA on ranks with Dunn's post hoc comparisons). Addition of latrunculin A, cytochalasin $\mathrm{B}$, or nocodazole plus latrunculin $\mathrm{A}(\mathrm{Noc}+\mathrm{Lat})$ decreased the F-actin-to-G-actin ratio in growth cones to levels statistically similar to those in the axon. All F-actin-to-G-actin ratios in the axon are statistically similar. Con, Control; Tax, taxol; Noc, nocodazole; Lat A, latrunculin A; Cyto B, cytochalasin B; $N+L$, nocodazole plus latrunculin. 


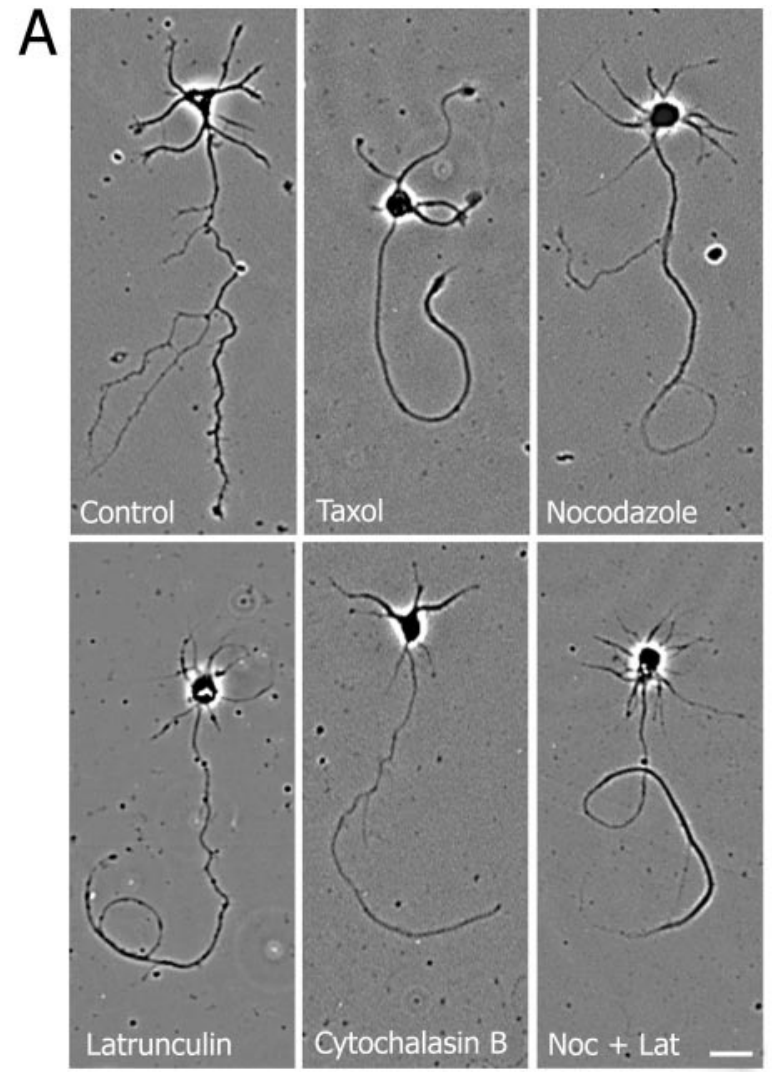

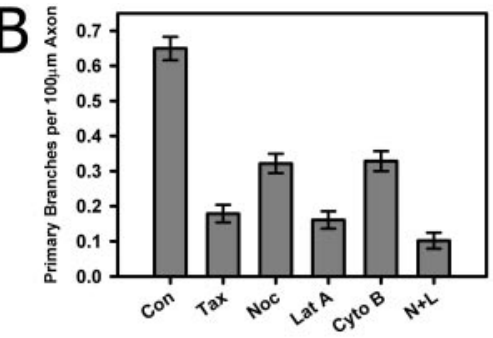
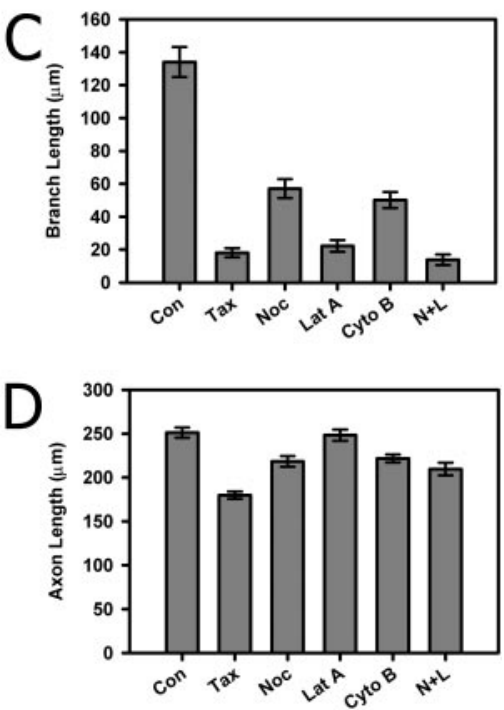

Figure 5. Inhibition of axon branching but not axon outgrowth after drug treatments that attenuate microtubule and actin dynamics. $A$, Examples of a control cortical neurons and those treated with 10 $\mathrm{nm}$ taxol, $33 \mathrm{~nm}$ nocodazole, $0.5 \mu \mathrm{m}$ latrunculin A, $1.0 \mu \mathrm{M}$ cytochalasin $\mathrm{B}$, or both nocodazole and latrunculin A cultured for $48 \mathrm{hr}$ and fixed. All drug treatments resulted in axons with curved trajectories and very few branches. $B, C$, Bar graphs showing that treatment with drugs that affect either microtubule or F-actin dynamics inhibit axon branching and reduce branch length (all treatments, $p<$ 0.01 compared with controls, KruskalWallis one-way ANOVA on ranks with Dunn's post hoc comparisons; $n=$ between 110 and 330 neurons for each treatment from 4 separate experiments). $D$, Bar graph showing that drug treatments do not significantly reduce axon length. Only neurons treated with taxol were significantly shorter than controls $(p<$ $0.05)$. All graphs are plotted as mean \pm SEM. Con, Control; Tax, taxol; Noc, nocodazole; Lat A, latrunculin A; Cyto B, cytochalasin $\mathrm{B} ; N+L$, nocodazole plus latrunculin. Scale bar, $20 \mu \mathrm{m}$. tween two objects in addition to the $0.6 \mu \mathrm{m}$ of possible movement and dynamics between the first and second acquisitions (1.3-1.8 sec between exposures of microtubules and F-actin; Waterman-Storer and Salmon, 1998). The area of microtubule-F-actin colocalization in living growth cones and branch points was measured by merging flat field-corrected 16 bit microtubule-F-actin images and then applying a threshold to the 24 bit color overlay images with the set color threshold command in Metamorph. The lamellipodium of the growth cone or axon branch point was traced, and the threshold area within the lamellipodium was measured for each time point. Movies of montaged images were compiled from time-lapse images of F-actin, images of microtubules, and merged images of microtubule-F-actin with the montage stacks function in Metamorph and saved as individual tiff images. These images were assembled into QuickTime movies (Premiere; Adobe Systems, Mountain View, CA). Image stacks were compressed $50 \%$ with the motion JPEG codec in Premiere. Images presented in the text were sharpened with the unsharp mask and low-pass filter functions in Metamorph and compiled as 8 bit gray scale or 24 bit color images (Adobe Photoshop). Graphs were constructed in SigmaPlot, and statistical analyses were performed with SigmaStat (Jandel Scientific, Corte Madera, CA).

\section{RESULTS}

\section{F-actin and microtubules reorganize during axon branching}

To determine how actin and microtubules reorganize during branching, we followed changes in the distribution of F-actin and microtubules during initiation of new growth from the growth cone and the axon shaft. We labeled fixed neurons at various stages of branching with fluorescent phalloidin and antibodies to tubulin. In smaller growth cones (Fig. $1 A$ ), which we have identified previously as rapidly growing (Szebenyi et al., 1998), the central region is occupied by straight bundles of microtubules as well as actin filaments. In the transition region between the central and peripheral regions of the growth cone (Forscher and Smith, 1988; Bridgman and Dailey, 1989), microtubules overlap with actin filaments and project outward into the lamellipodium.
In the peripheral lamellipodium, actin filaments predominate. Within filopodia they form straight bundles that extend proximally into the lamellipodium. In large growth cones, which we have found to undergo prolonged pausing before development of branches or reorganization into a new axon, microtubules in the central region are organized in a prominent loop (Dent et al., 1999) from which F-actin is excluded (Fig. 1B).

To observe dynamic changes in F-actin and microtubules during successive stages in the development of axon branches, we microinjected living neurons simultaneously with fluorescent phalloidin and tubulin and imaged axons and growth cones for 5-28 hr at intervals of $30 \mathrm{~min}$ to $2 \mathrm{hr}$ with time-lapse fluorescence microscopy. Growth cone morphologies and distributions of F-actin and microtubules were similar to those observed in fixed neurons, showing that the injection and imaging procedures were not injurious to the neurons. During imaging over 5-28 hr, neurons continued to extend neurites that grew and branched in a manner similar to uninjected neurons. The use of phalloidin, which can potentially stabilize actin filaments, did not interfere with the ability of actin to reorganize over time (Sanders and Wang, 1991; O'Connor and Bentley, 1993; Lin and Forscher, 1995; Waterman-Storer et al., 2000). In large paused growth cones ( $n=26$ from seven separate experiments), F-actin was excluded from the central region. As we have shown previously (Dent et al., 1999), selective regions of the microtubule loop splay apart and give rise to short microtubule fragments that invade the lamellipodium in locations where a new axon or a branch will eventually extend. These fragments are indeed short microtubules capable of independent movements rather than longer microtubules connected to the central microtubule loop. We determined this in both live and fixed cells by focusing through the entire depth of the growth cone (Dent et al., 1999). As shown in 

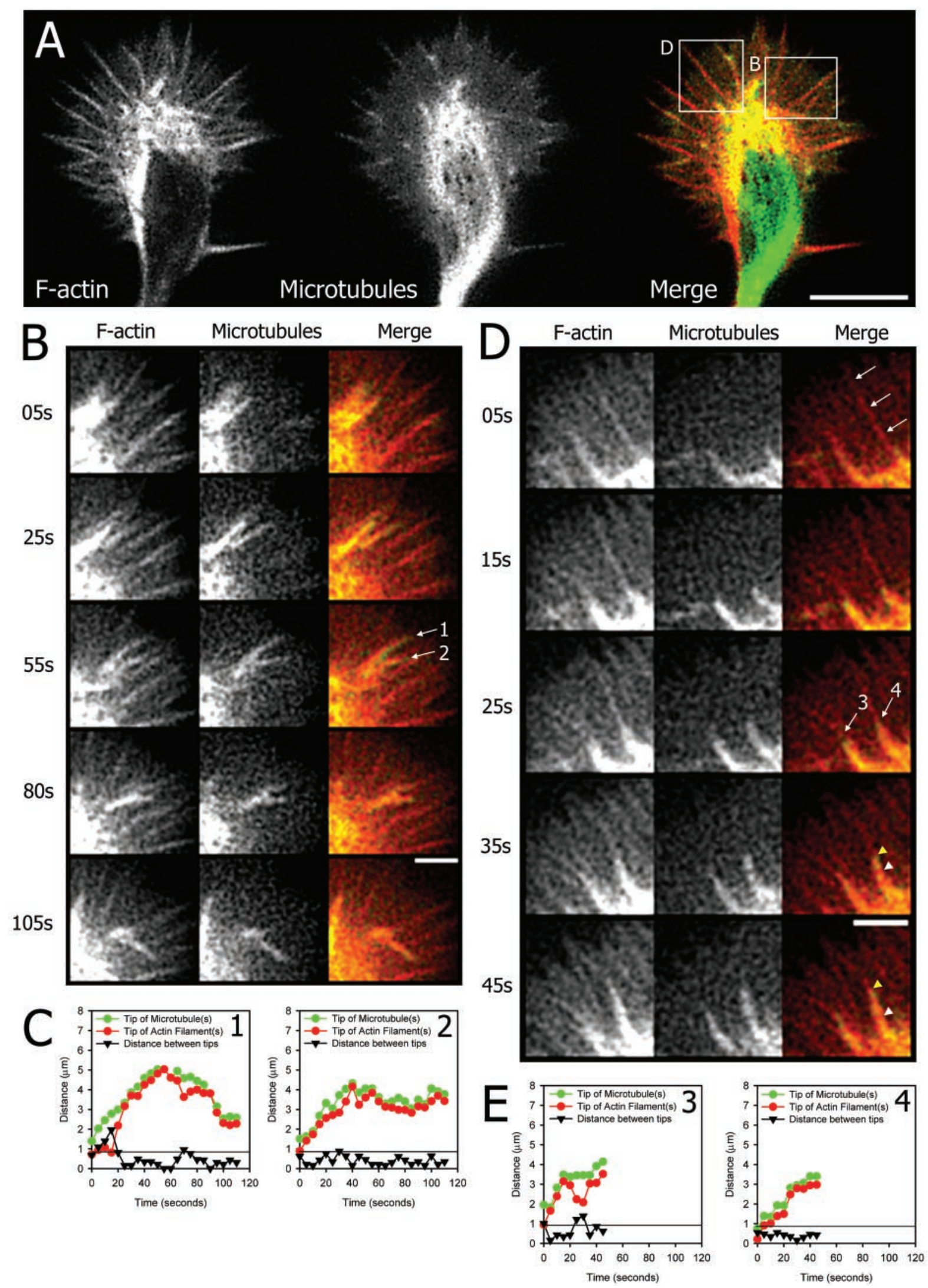

Figure 6. Tandem polymerization and depolymerization of microtubules and F-actin in growth cones. $A$, Images of the same living growth cone at a single time point showing location of actin filaments at left, microtubules in the middle, and a merged image at right. In the merged image, F-actin is pseudocolored red, microtubules green, and their colocalization in the transition region yellow. B, Higher-power images taken from the boxed region in $A$ (at right) showing that actin filaments and microtubules polymerize and depolymerize together. From $05 s$ to $55 s$, microtubules and actin filaments polymerize outward and reach the periphery of the growth cone. From $80 \mathrm{~s}$ to $105 \mathrm{~s}$, one microtubule and associated bundle (Figure legend continues) 
Figure $1 C$, F-actin selectively accumulates in and overlaps with microtubules in these regions of the loop. F-actin and microtubules continue to overlap in the transition region for many hours until a definitive new axon, tipped by a small growth cone, has emerged from the large paused growth cone (Fig. 1C, 7:47hr and 26:32hr).

Similar reorganization of the F-actin and microtubule cytoskeleton occurs during formation of branches from remnants of paused growth cones on the axon shaft. Lengthy imaging sequences ( $n=26$ sequences, 34 branches) show that branches often begin as a single filopodium containing F-actin and microtubules (Fig. $2 A, B$ ). At branch points along the axon shaft (Fig. $2 C, D)$, bundled microtubules splay apart, coincident with an accumulation of F-actin, and microtubules invade the newly forming branch (Fig. 2D). The growth cone at the tip of the branch has high levels of F-actin that overlap with microtubules, whereas F-actin has disappeared from proximal regions of the branch. In living growth cones, microtubules and F-actin appear to be closely apposed. We confirmed this with electron microscopy of fixed cortical growth cones (Fig. $2 E$ ), which showed that microtubules splaying from the central loop (Fig. 2E, inset) extend into the lamellipodium, where they are closely apposed to bundles of actin filaments (Dailey and Bridgman, 1991; Rochlin et al., 1999). These results show that development of branches from a large paused growth cone or from its remnants along the axon shaft is accompanied by local accumulation of F-actin, which coincides with splaying and fragmentation of bundled microtubules.

\section{Attenuation of microtubule dynamics or actin polymerization inhibits axon branching}

The colocalization of F-actin with microtubules suggests that they interact during formation of axon branches. Dynamic microtubules are capable of growth and shortening through cycles of polymerization and depolymerization (Mitchison and Kirschner, 1984; Desai and Mitchison, 1997) and previous studies have shown the importance of dynamic microtubules in growth cone turning at inhibitory boundaries (Tanaka et al., 1995; Williamson et al., 1996; Challacombe et al., 1997). To determine whether F-actin-microtubule interaction is necessary for branch formation, we used drug treatments that selectively inhibited cytoskeletal dynamics. We treated cortical cultures with drugs (taxol and nocodazole) that at low concentrations attenuate microtubule dynamics (Vasquez et al., 1997; Mikhailov and Gundersen, 1998; Yvon et al., 1999; Kabir et al., 2001). To inhibit actin polymerization, we used latrunculin $\mathrm{A}$, which sequesters actin monomers, or cytochalasin B, which severs actin filaments and caps their barbed ends (for review, see Spector et al., 1999). We first determined how these drugs affect the distribution of microtubules and F-actin. It is known that older, more stable microtubules are more likely to be acetylated, whereas newly formed microtubules are more likely to be tyrosinated (Brown et al., 1993). Because newly polymerized microtubules are dynamic (Baas and Black, 1990; Li and Black, 1996), staining for tyrosinated microtubules generally reflects the dynamic population. Staining of fixed cortical neurons with antibodies to acetylated tubulin showed that this population is contained throughout the axon shaft, in the bundles of looped microtubules within the central region of the growth cone, and in the proximal region of developing axon branches (Fig. $3 A, B$ ). In contrast, microtubules in distal regions of developing branches and in the transition region of the growth cone are tyrosinated. Furthermore, all of the microtubule fragments present in the distal regions of growth cones were tyrosinated (data not shown). Importantly, the location of dynamic tyrosinated microtubules coincides with regions of high $\mathrm{F}$-actin, whereas regions of older acetylated microtubules contain little F-actin (Fig. $3 A, B$ ). Treatment of cortical cultures with taxol and nocodazole causes the disappearance of many but not all of the dynamic tyrosinated microtubules from the transition zone, whereas the acetylated microtubules are unaffected (Fig. 3C-E). As expected, drugs that attenuate microtubule dynamics decreased the length of tyrosinated microtubule ends (Fig. 4A; Rochlin et al., 1996). Surprisingly, F-actin-depolymerizing drugs also decreased the length of tyrosinated microtubule ends (Fig. 4A). Similarly, F-actin concentration in the growth cone, as measured by a ratio of fluorescent F-actin against fluorescent G-actin, is reduced not only by latrunculin A and cytochalasin B but also by nocodazole (Fig. $4 B)$. Neurons treated with taxol and nocodazole had axons that were similar in length to controls (Fig. $5 A, D$ ) but had torturous trajectories and only a few branches (Fig. $5 B$ ), which were significantly shorter than controls (Fig. $5 C$ ). Treatment of cortical neurons with latrunculin $\mathrm{A}$, cytochalasin $\mathrm{B}$, or a combination of nocodazole and latrunculin A had effects similar to taxol and nocodazole in reducing axon branching. Thus, treatment of cortical neurons with reagents that inhibit polymerization of actin filaments, microtubules, or both selectively inhibit initiation of axon branches without significantly reducing axon length. These results suggest that although axon outgrowth can proceed with attenuation of either dynamic microtubules or actin polymerization, initiation of axon branching requires both dynamic microtubules and actin filaments.

\section{F-actin-microtubule interactions involve copolymerization}

To determine the nature of F-actin-microtubule interactions in living growth cones and at axon branch points, we used rapid acquisition $(5-15 \mathrm{sec})$ of closely spaced (1-2 sec) sequential images of microtubules and actin filaments $(n=36$ sequences in six growth cones and five branch points) over periods of $10-20$ min. We chose large paused growth cones to visualize cytoskeletal interactions associated with branching (Figs. 1, 2). Movies of sequential images of actin filaments and microtubules allowed us to analyze frame by frame the reorganization of each cytoskeletal

\section{$\longleftarrow$}

(Figure legend continued.) of F-actin (1) depolymerizes and moves rearward with the retrograde actin flow, whereas the other microtubule and associated

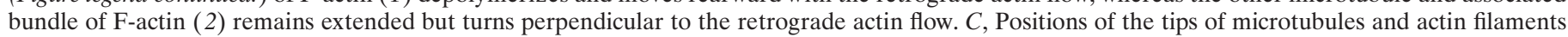

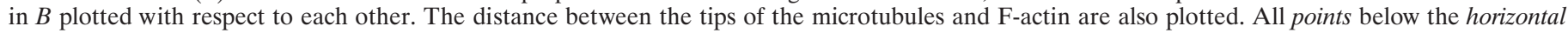

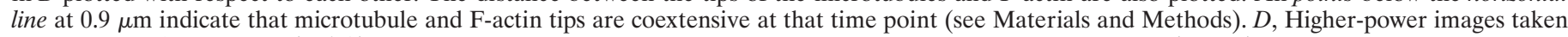

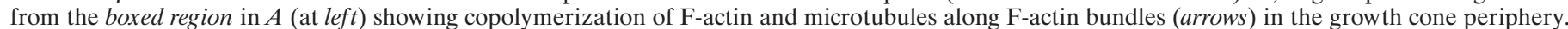

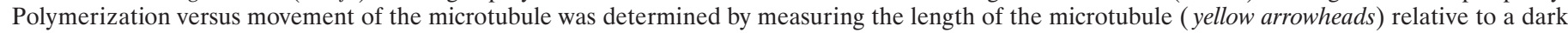

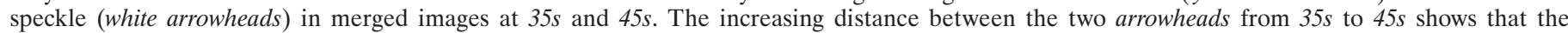

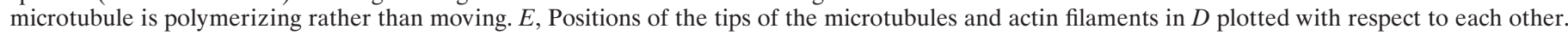
Scale bars: $A, 10 \mu \mathrm{m} ; B, D, 3 \mu \mathrm{m}$. 

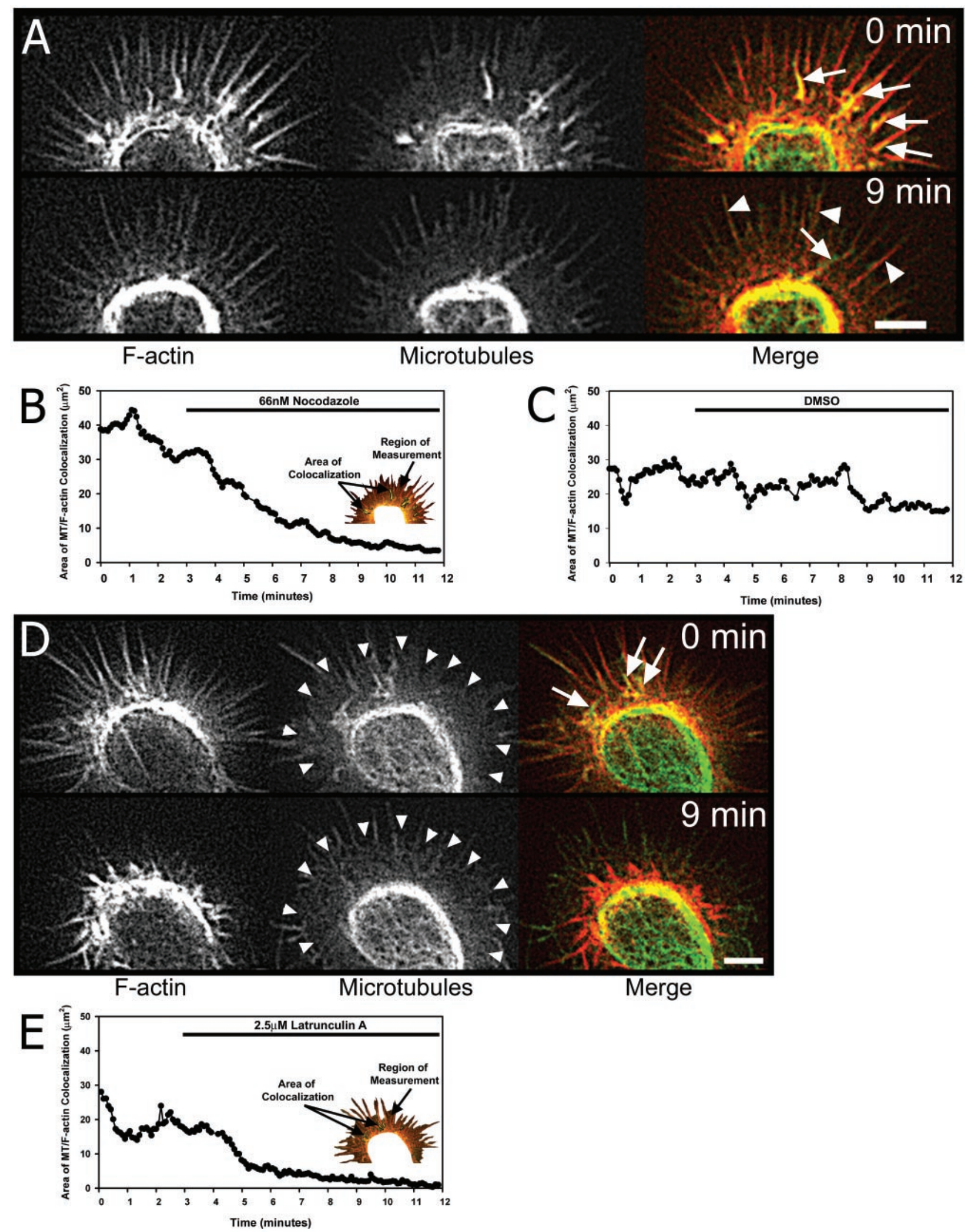

Figure 7. Attenuation of microtubule and actin dynamics reduces F-actin-microtubule colocalization in the growth cone transition region. $A$, Time-lapse images showing movements of actin filaments (left), dynamic microtubules (middle), and merged images (right) in a growth cone lamellipodium. Arrows (merged image, $0 \mathrm{~min}$ ) point to examples of closely associated microtubules and actin filaments. Numbers refer to time in minutes. Addition of $66 \mathrm{~nm}$ nocodazole at 3 min attenuates microtubule dynamics (4-9 min), and microtubules and associated actin filaments are brought rearward in retrograde actin flow. Few microtubules and associated actin filaments re-extend into the lamellipodium (Figure legend continues) 
element separately and then combine the images as color overlaysto analyze how F-actin and microtubules interact. It is known that F-actin forms straight stiff bundles in filopodia and a meshwork in lamellipodia (Bridgman and Dailey, 1989; Lewis and Bridgman, 1992). In both regions of the growth cone, actin filaments polymerize at their distal ends and depolymerize proximally. Actin filaments in both regions undergo continuous retrograde flow (Forscher and Smith, 1988; Welnhofer et al., 1997; Mallavarapu and Mitchison, 1999). Previous studies have shown that filamentous actin in the transition region of the growth cone can take more sinuous forms termed "intrapodia" that polymerize outward and protrude into the lamellipodium (Katoh et al., 1999; Rochlin et al., 1999). As shown in one example of a large paused growth cone in Figure $6 A$, actin filaments, resembling intrapodia, polymerize from the transition region outward into the periphery of the lamellipodium and depolymerize back toward the transition region (Fig. 6). Sometimes this intrapodial $\mathrm{F}$-actin follows the straight trajectories of F-actin bundles in filopodia (Fig. 6D; Sider et al., 1999; Waterman-Storer et al., 2000). In most cases, intrapodial F-actin becomes orientated tangentially to filopodia and is then dragged backward in the retrograde actin flow. Often intrapodial F-actin depolymerizes as it collapses back onto the microtubule loop (Fig. 6B). Surprisingly, in corresponding images, microtubules appear to follow the same trajectories as intrapodial actin filaments. As seen in the movies of Figure $6 A$ (available at http://kalil.anatomy.wisc.edu), the overall impression is that of sinusoidal movement of F-actin and microtubules extending outward from the transition region of the growth cone into the lamellipodium (Fig. $6 A$; see supplemental movies). The merged images in Figure $6, B$ and $D$, show that actin filaments and microtubules change their positions together by polymerizing and depolymerizing in tandem (see supplemental movies of Fig. $6 B, D)$. We confirmed this by plotting the locations of their distal tips independently (Fig. 6C,E). To determine whether these positional changes could involve microtubule polymerization, we measured growth and shrinkage at their tips using the technique of fluorescent speckle microscopy (Waterman-Storer et al., 1998), in which intermittent incorporation of low concentrations of fluorescent tubulin into microtubules results in speckles, which serve as fiduciary marks on the microtubules (Fig. 6D). We found that microtubules polymerize and depolymerize at rates averaging $7.48 \pm 0.41$ and $6.98 \pm 0.75$ $\mu \mathrm{m} / \mathrm{min}$, respectively ( $n=31$ microtubules from six growth cones; mean \pm SEM), similar to observations in Aplysia growth cones (Kabir et al., 2001). Almost invariably, whenever microtubules polymerized, actin filaments copolymerized with them (94\%; $n=31$ microtubules and associated actin filaments). Taken together, these results show that microtubules can interact with actin filaments by polymerizing and depolymerizing together.

\section{F-actin-microtubule interactions are essential for branch formation}

Application of cytoskeletal depolymerizing drugs showed that attenuation of dynamic microtubules or depolymerization of F-actin severely reduces initiation of axon branches. To demonstrate directly how effects of these drugs on the cytoskeleton lead to inhibition of axon branching, we applied either nocodazole or latrunculin A to living neurons and imaged dynamic changes in microtubules and actin filaments in growth cones $(n=10)$ and at axon branch points ( $n=3$; see supplemental movies). Within several minutes of application of nocodazole (Fig. $7 A$ ), outward growth of dynamic microtubules in the growth cone was inhibited. This treatment also inhibited extension of actin filaments into the lamellipodium (Fig. 7A). Application of latrunculin A inhibited actin polymerization and concomitantly prevented outward growth of microtubules into the growth cone lamellipodium (Fig. $7 D$ ). Measurements of the area of microtubule-F-actin colocalization in the growth cone lamellipodium showed that colocalization declined sharply within minutes of drug application (Fig. $7 B, E$ ) but not in control experiments (Fig. 7C). During the period studied, retrograde actin flow, measured by rates of movement of phalloidin speckling, was not disrupted by treatment with nocodazole $(6.81 \pm 0.27 \mu \mathrm{m} / \mathrm{min}$ before and $6.88 \pm 0.33 \mu \mathrm{m} / \mathrm{min}$ after; $n=5)$ or latrunculin A $(5.42 \pm 0.48 \mu \mathrm{m} / \mathrm{min}$ before and $4.93 \pm$ $0.19 \mu \mathrm{m} / \mathrm{min}$ after; $n=5$ ). Loss of polymerization caused associated F-actin and microtubules to be carried rearward in the retrograde actin flow and to collapse back onto the microtubule loop in the central region of the growth cone (Fig. $7 A, D$; see supplemental movies). By the end of the image sequences, Factin-microtubule polymerization into the lamellipodium was almost completely inhibited, whereas the microtubule loop in the central region of the growth cone was unaffected. As shown in Figure 8, nocodazole and latrunculin A produced similar effects at axon branch points such that microtubules and associated F-actin collapsed rearward onto the bundled microtubules in the axon shaft (see supplemental movies). Latrunculin A, unlike nocodazole, also eliminated polymerization of F-actin in the peripheral region of the growth cone and at axon branch points. However, in both regions, loss of cytoskeletal dynamics did not cause collapse of the lamellipodium during the imaging periods, showing that cytoskeletal changes were not simply attributable to collapse of the membrane (Figs. 7D, 8D). For both drugs, the net result of decreases in actin and microtubule polymerization was a decrease in the region of F-actin-microtubule colocalization in growth cones as well as at axon branch points (Figs. 7, 8). The region of colocalization decreased by $61 \pm 3 \%(n=5)$ for nocodazole and by $74 \pm 1 \%(n=5)$ for latrunculin A by $5 \mathrm{~min}$ after application of the drug. Taken together, these results demonstrate directly that inhibition of either F-actin or microtubule

\section{$\leftarrow$}

(Figure legend continued.) (arrow at 9 min), but F-actin bundles in filopodia remain (arrowheads at 9 min). Microtubules are pseudocolored green, actin

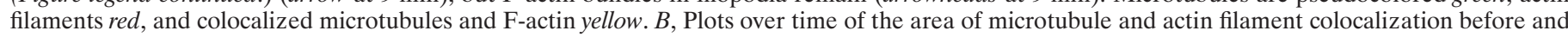

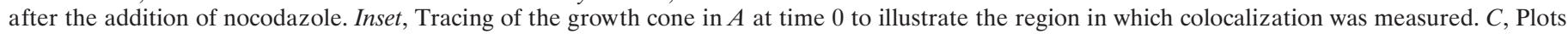

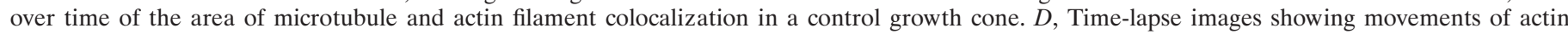

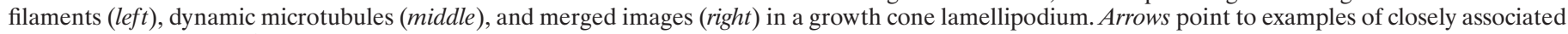

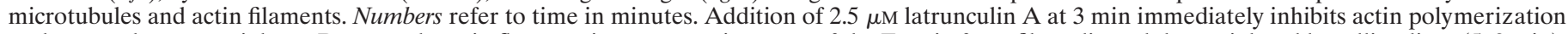

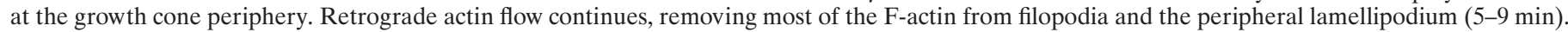

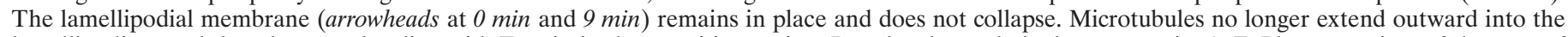

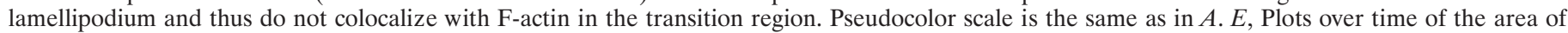

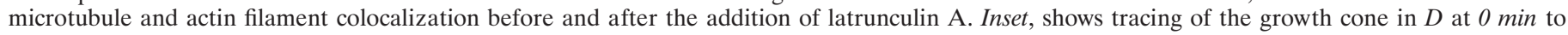
illustrate the region in which colocalization was measured. Scale bars, $5 \mu \mathrm{m}$. 


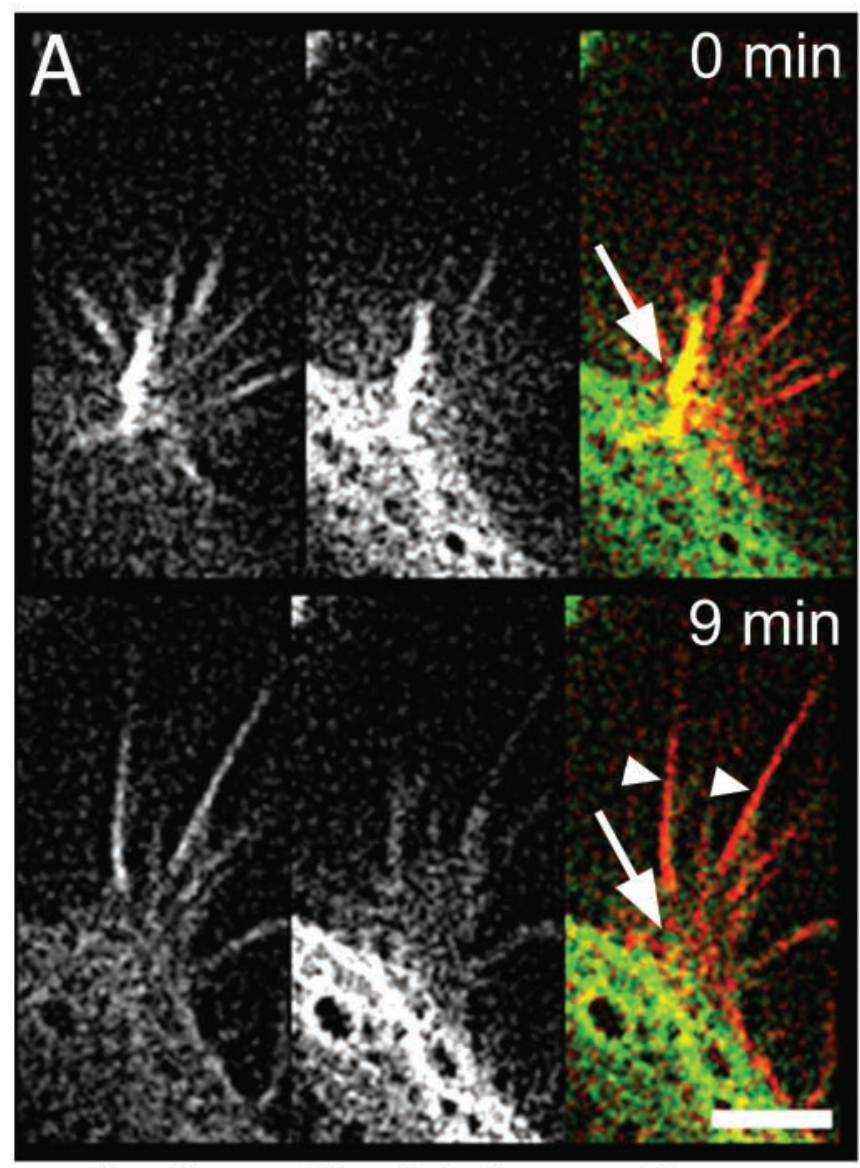

F-actin Microtubules

Merge
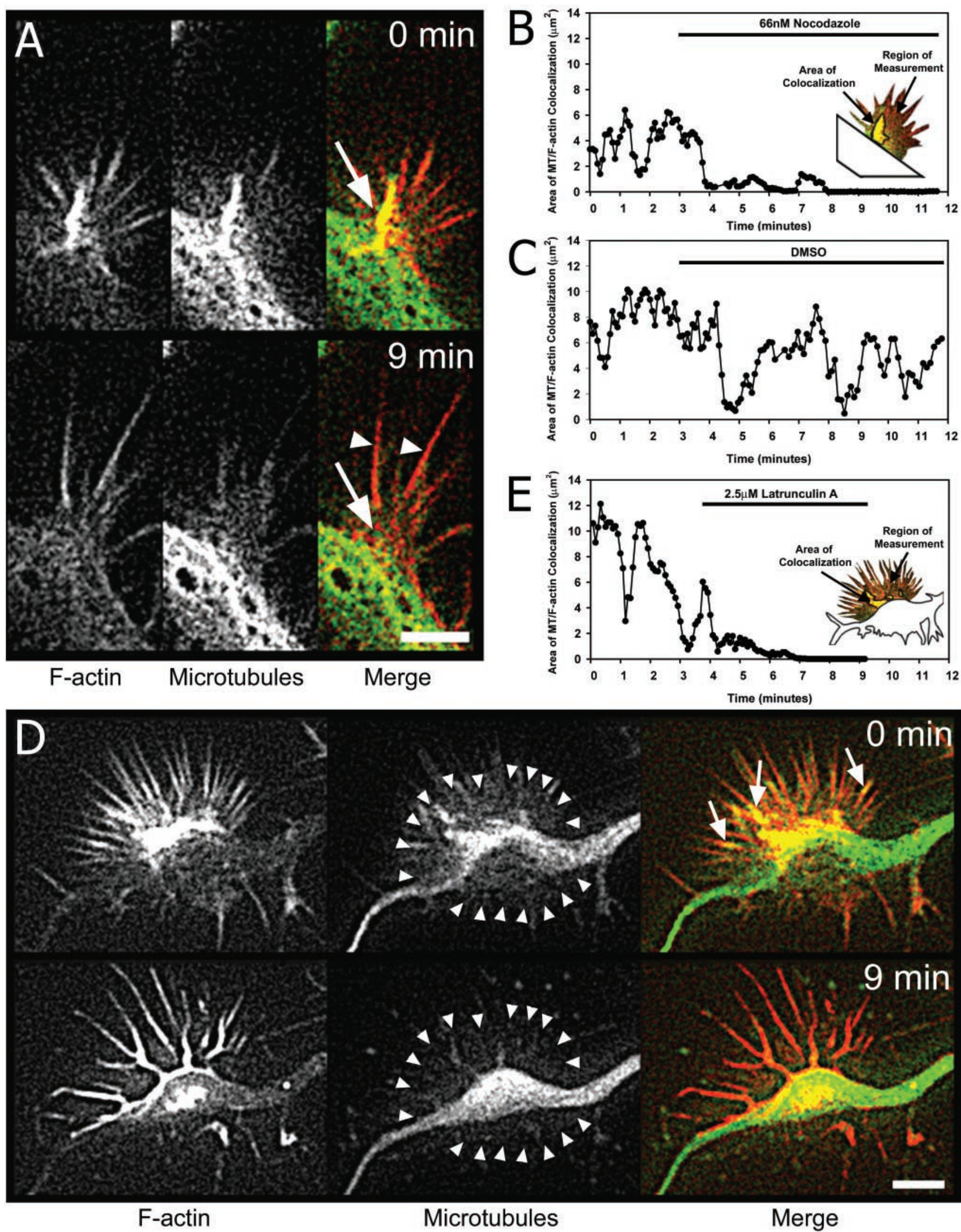

$9 \mathrm{~min}$

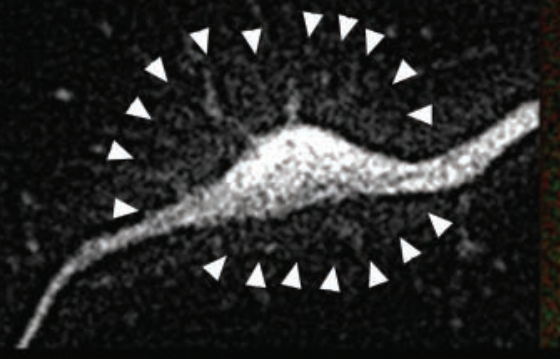

Microtubules

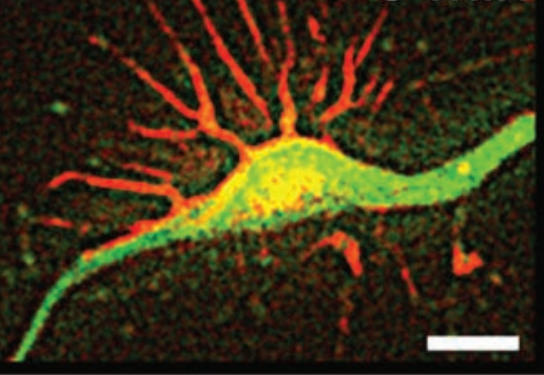

Merge

Figure 8. Attenuation of microtubule and actin dynamics reduces F-actin-microtubule colocalization at axon branch points. $A$, Time-lapse images showing movements of actin filaments (left), dynamic microtubules (middle), and merged images (right) at a branch point on the axon shaft. Arrows (merged image, $0 \mathrm{~min}$ ) point to examples of closely associated microtubules and actin filaments. Numbers refer to time in minutes. Addition of 66 nM nocodazole at $3 \mathrm{~min}$ attenuates microtubule dynamics (4-9 $\mathrm{min})$, and microtubules and associated actin filaments are brought rearward in retrograde actin flow. By $9 \mathrm{~min}$, microtubules and F-actin no longer colocalize in the transition of the lamellipodium (arrow at 9 min), but (Figure legend continues) 
dynamics also inhibits the dynamics of both cytoskeletal elements and leads to inhibition of directed growth. This suggests that interaction between dynamic microtubules and actin filaments is essential for initiation of axon branching.

\section{DISCUSSION}

In this study we observed directly the cytoskeletal reorganization underlying cortical axon branching. We found that branching from the growth cone and the axon shaft is always preceded by splaying apart of looped or bundled microtubules which is accompanied by localized accumulation of F-actin. Dynamic microtubules colocalize with F-actin in transition regions of growth cones and axon branch points, consistent with observations in fixed growth cones (Bridgman and Dailey, 1989; Tanaka et al., 1995; Challacombe et al., 1996, 1997; Williamson et al., 1996; Rochlin et al., 1999), whereas F-actin is excluded from regions of stable microtubules (Fig. 9). Interactions between microtubules and actin filaments involve coordinated polymerization and depolymerization. Drugs that attenuate either microtubule or actin dynamics concomitantly abolish microtubule-F-actin interactions at the growth cone and at axon branch points. Importantly, these drug treatments inhibit axon branching but not axon elongation, demonstrating that interactions between dynamic microtubules and actin filaments are essential for initiating axon growth in new directions.

\section{F-actin-microtubule interactions in neuronal growth cones}

Previous time-lapse imaging studies have shown that changes in the distribution and orientation of microtubules and actin filaments underlie changes in the direction of axonal growth. This has been demonstrated in situ at decision regions in the grasshopper limb bud (Sabry et al., 1991; O’Connor and Bentley, 1993) and in growth cones of Aplysia neurons interacting with a cellular target or pseudotarget (Lin and Forscher, 1993, 1995; Suter et al., 1998), where microtubules reorient toward focal concentrations of F-actin. Reorganization of microtubules from bundled to splayed forms in regions of high F-actin has also been documented at axon branch points of fixed dorsal root ganglion neurons (Gallo and Letourneau, 1998). Several models for F-actinmicrotubule interactions have been proposed as a mechanism for axon guidance. Microtubules may be directed toward regions of attenuated F-actin flow associated with growth cone-target interactions (Lin et al., 1994; Suter and Forscher, 2000), or dynamic microtubule ends may be captured by actin filaments during growth cone turning (for review, see Bentley and O'Connor, 1994; Tanaka and Sabry, 1995). Our observations suggest that microtubules continually explore the growth cone periphery without attenuation of retrograde flow and, furthermore, that F-actin and microtubules are coextensive in the transition region. Thus, during directed axon outgrowth, the mode of F-actin-microtu-

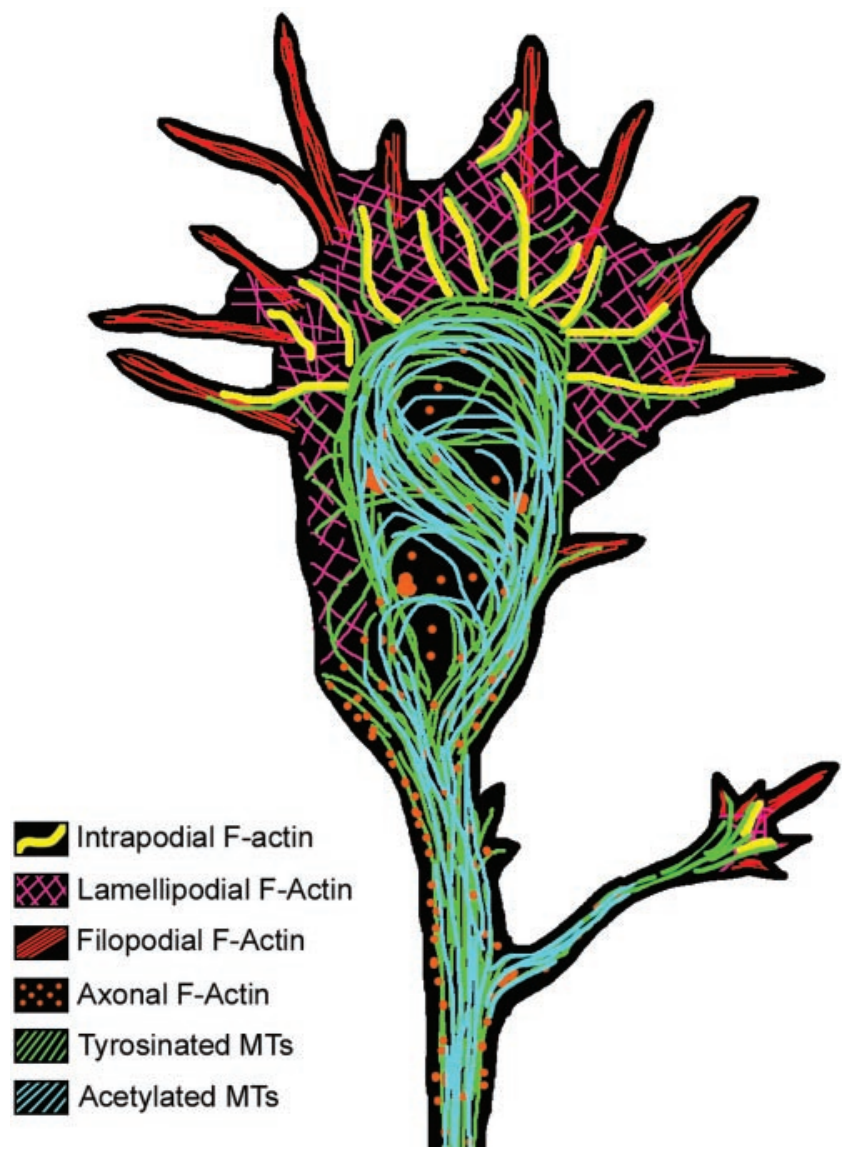

Figure 9. Summary schematic showing locations of microtubule and actin filament populations in a large paused growth cone and developing axon branch. Both tyrosinated and acetylated microtubules are located in the axon shaft and the central region of the growth cone. Tyrosinated microtubules extend into the lamellipodium, filopodia, and distal regions of axon branches. Tyrosinated microtubules colocalize with intrapodial actin filaments. F-actin in the axon shaft and central region of the growth cone forms dot-like structures that colocalize with acetylated microtubules.

bule interaction that we favor involves copolymerization rather than capturing mechanisms (Gordon-Weeks, 1991) or polymerization of microtubules into regions of attenuated retrograde F-actin flow (Suter et al., 1998; Suter and Forscher, 2000). For branches to form from the growth cone or the axon shaft, microtubules must be selectively stabilized in the preferred directions of growth (Liao et al., 1999). Previous studies in Aplysia growth cones (Forscher and Smith, 1988) showed that by $30 \mathrm{~min}$ after depolymerization of actin filaments by treatment with cytochala$\sin \mathrm{B}$, microtubules in the central region invade the growth cone periphery from which they are normally excluded presumably by

\section{$\leftarrow$}

(Figure legend continued.) F-actin bundles in filopodia remain (arrowheads at 9 min). Microtubules are pseudocolored green, actin filaments red, and colocalized microtubules and F-actin yellow. $B$, Plots over time of the area of microtubule and actin filament colocalization before and after the addition of nocodazole. Inset, Tracing of the branch point in $A$ at $0 \mathrm{~min}$ to illustrate the region in which colocalization was measured. $C$, Plots over time of the area of microtubule and actin filament colocalization in a branch point from a control neuron. $D$, Time-lapse images showing movements of actin filaments (left), dynamic microtubules (middle) and merged images (right) at a branch point on the axon shaft. Arrows point to examples of closely associated microtubules and actin filaments. Numbers refer to time in minutes. Addition of $2.5 \mu \mathrm{M}$ latrunculin A at 4 min immediately inhibits actin polymerization in the lamellipodium. Retrograde actin flow continues, removing most of the F-actin from the peripheral lamellipodium (5-9 min). The lamellipodial membrane (arrowheads at $0 \mathrm{~min}$ and $9 \mathrm{~min}$ ) remains in place and does not collapse. Microtubules no longer extend outward into the lamellipodium and thus do not colocalize with F-actin in the transition region at the branch point. Pseudocolor scale is the same as in $A$. $E$, Plots over time of the area of microtubule and actin filament colocalization before and after the addition of latrunculin A. Inset, Tracing of the branch point in $D$ at 0 min to illustrate the region in which colocalization was measured. Scale bars, $5 \mu \mathrm{m}$. 
the presence of actin. Our results show that depolymerization of actin filaments causes microtubules to retreat from the periphery toward the central region within $10 \mathrm{~min}$. However, we did observe that at later times (Fig. 4), many microtubules are present in the periphery, consistent with results in Aplysia.

\section{Coordinated polymerization of microtubules and actin filaments}

Previous studies have suggested that actin filaments and microtubules can each influence the polymerization of the other either directly or at a distance (for review, see Waterman-Storer and Salmon, 1999; Goode et al., 2000). For example, in fibroblasts, drug treatments that activate microtubule polymerization concomitantly induce actin polymerization and activation of Rac1 at the periphery (Waterman-Storer et al., 1999). Other proteins, such as Cdc-42-interacting-protein-4 (Tian et al., 2000) and RhoG (Ren et al., 1999), have been proposed as part of a pathway linking the polymerization of actin and microtubules. Although such pathways have not been studied in neurons, microtubule polymerization in growth cones of sympathetic neurons has been shown to activate formation of actin-based protrusive structures (intrapodia; Rochlin et al., 1999) that develop in the transition region. These are distinct from the more stable actin bundles in filopodia (Mallavarapu and Mitchison, 1999) and the meshwork of F-actin in lamellipodia. It seems likely that the dynamic actin filaments in the transition region of cortical growth cones are intrapodia. This is consistent with our observations that copolymerization is the major form of interaction between F-actin and dynamic microtubules in the transition region.

Copolymerization would require signaling between actin filaments and microtubules at the growth cone and at axon branch points. We have shown that attenuation of either F-actin or microtubule dynamics eliminates their copolymerization and thereby abolishes initiation of axon branching. Interaction between dynamic actin filaments and microtubules is required for directed axon growth from the growth cone and from the axon shaft in the form of branches but is not required for axon extension per se (Fig. 5). This suggests that bidirectional signaling between F-actin and microtubules is necessary for coordinating their polymerization in preferred directions of growth. Bidirectional signaling between dynamic microtubules and intrapodial actin filaments could be accomplished by selective binding and release of specific proteins that affect the polymerization of the other cytoskeletal element (for review, see McNally, 2001; Schuyler and Pellman, 2001). Furthermore, our data suggest that the ability of microtubules to bind certain proteins may depend on the degree of tubulin tyrosination. Later, once axon branches are formed, their further growth is dominated by transport of microtubules into the growing branch (Dent et al., 1999; Gallo and Letourneau, 1999).

Regulation of cytoskeletal polymerization has implications for many aspects of axon outgrowth. For example, regulation of assembly and disassembly of microtubule loops has been shown to affect growth (Tanaka et al., 1995) and stabilization of the growth cone (Dent et al., 1999) as well as formation of presynaptic boutons (Roos et al., 2000). Actin polymerization, under the control of various regulatory proteins (Lanier and Gertler, 2000), has been found to influence the speed of growth cone advance (Dent and Meiri, 1992; Kuhn et al., 1998; Brown et al., 2000) and concomitantly the innervation of targets by axon branches (Wills et al., 1999). At present, we do not understand the mechanisms of microtubule looping during growth cone pausing or microtubule splaying and fragmentation during transitions to new growth. One possibility is that these changes in the organization of microtubules are regulated by bidirectional interactions with dynamic actin filaments. Pausing or slowing of growth cone advance is closely related to induction of axon branches (Szebenyi et al., 1998) through the action of target derived factors such as fibroblast growth factor 2 (Szebenyi et al., 2001). Other inhibitory guidance molecules may also inhibit growth cone advance and thus promote axon branching. At present, the intracellular pathways linking target-derived guidance cues to the cytoskeleton are not well understood. However, interaction between F-actin and microtubules implies that factors that influence the dynamic state of either F-actin or microtubules may affect both. In future studies it will therefore be important to elucidate the signaling pathways that regulate microtubule-F-actin interactions and to identify the proteins that coordinate their polymerization and depolymerization.

\section{REFERENCES}

Baas PW, Black MM (1990) Individual microtubules in the axon consist of domains that differ in both composition and stability. J Cell Biol 111:495-509.

Bentley D, O'Connor TP (1994) Cytoskeletal events in growth cone steering. Curr Opin Neurobiol 4:43-48.

Bridgman PC, Dailey ME (1989) The organization of myosin and actin in rapid frozen nerve growth cones. J Cell Biol 108:95-109.

Brown A, Li Y, Slaughter T, Black MM (1993) Composite microtubules of the axon: quantitative analysis of tyrosinated and acetylated tubulin along individual axonal microtubules. J Cell Sci 104:339-352.

Brown MD, Cornejo BJ, Kuhn TB, Bamburg JR (2000) Cdc42 stimulates neurite outgrowth and formation of growth cone filopodia and lamellipodia. J Neurobiol 43:352-364.

Challacombe JF, Snow DM, Letourneau PC (1996) Actin filament bundles are required for microtubule reorientation during growth cone turning to avoid an inhibitory guidance cue. J Cell Sci 109:2031-2040.

Challacombe JF, Snow DM, Letourneau PC (1997) Dynamic microtubule ends are required for growth cone turning to avoid an inhibitory guidance cue. J Neurosci 17:3085-3095.

Dailey ME, Bridgman PC (1991) Structure and organization of membrane organelles along distal microtubule segments in growth cones. J Neurosci Res 30:242-258.

Dent EW, Meiri KF (1992) GAP-43 phosphorylation is dynamically regulated in individual growth cones. J Neurobiol 23:1037-1053.

Dent EW, Callaway JL, Szebenyi G, Baas PW, Kalil K (1999) Reorganization and movement of microtubules in axonal growth cones and developing interstitial branches. J Neurosci 19:8894-8908.

Desai A, Mitchison TJ (1997) Microtubule polymerization dynamics. Annu Rev Cell Dev Biol 13:83-117.

Forscher P, Smith SJ (1988) Actions of cytochalasins on the organization of actin filaments and microtubules in a neuronal growth cone. J Cell Biol 107:1505-1516.

Gallo G, Letourneau PC (1998) Localized sources of neurotrophins initiate axon collateral sprouting. J Neurosci 18:5403-5414.

Gallo G, Letourneau PC (1999) Different contributions of microtubule dynamics and transport to the growth of axons and collateral sprouts. J Neurosci 19:3860-3873.

Goode BL, Drubin DG, Barnes G (2000) Functional cooperation between the microtubule and actin cytoskeletons. Curr Opin Cell Biol 12:63-71.

Gordon-Weeks PR (1991) Evidence for microtubule capture by filopodial actin filaments in growth cones. NeuroReport 10:573-576.

Halloran MC, Kalil K (1994) Dynamic behaviors of growth cones extending in the corpus callosum of living cortical brain slices observed with video microscopy. J Neurosci 14:2161-2177.

Hyman A, Drechsel D, Kellogg D, Salser S, Sawin K, Steffen P, Wordeman L, Mitchison T (1991) Preparation of modified tubulins. Methods Enzymol 196:478-485.

Kabir N, Schaefer AW, Nakhost A, Sossin WS, Forscher P (2001) Protein kinase $\mathrm{c}$ activation promotes microtubule advance in neuronal growth cones by increasing average microtubule growth lifetimes. J Cell Biol 152:1033-1044.

Kalil K, Szebenyi G, Dent EW (2000) Common mechanisms underlying growth cone guidance and axon branching. J Neurobiol 44:145-158.

Katoh K, Hammar K, Smith PJ, Oldenbourg R (1999) Birefringence imaging directly reveals architectural dynamics of filamentous actin in living growth cones. Mol Biol Cell 10:197-210.

Keating TJ, Peloquin JG, Rodionov VI, Momcilovic D, Borisy GG 
(1997) Microtubule release from the centrosome. Proc Natl Acad Sci USA 94:5078-5083.

Kuhn TB, Brown MD, Bamburg JR (1998) Rac1-dependent actin filament organization in growth cones is necessary for beta1-integrinmediated advance but not for growth on poly-D-lysine. J Neurobiol 37:524-540.

Lanier LM, Gertler FB (2000) From Abl to actin: Abl tyrosine kinase and associated proteins in growth cone motility. Curr Opin Neurobiol 10:80-87.

Lewis AK, Bridgman PC (1992) Nerve growth cone lamellipodia contain two populations of actin filaments that differ in organization and polarity. J Cell Biol 119:1219-1243.

Li Y, Black MM (1996) Microtubule assembly and turnover in growing axons. J Neurosci 16:531-544.

Liao G, Dreitzer G, Cook TA, Gundersen GG (1999) A signal transduction pathway involved in microtubule-mediated cell polarization. FASEB J [Suppl] 2:S257-S260.

Lin CH, Forscher P (1993) Cytoskeletal remodeling during growth cone-target interactions. J Cell Biol 121:1369-1383.

Lin CH, Forscher P (1995) Growth cone advance is inversely proportional to retrograde F-actin flow. Neuron 14:763-771.

Lin CH, Thompson CA, Forscher P (1994) Cytoskeletal reorganization underlying growth cone motility. Curr Opin Neurobiol 4:640-647.

Lubbers DW, Baumgartl H, Zimelka W (1994) Heterogeneity and stability of local $\mathrm{PO}_{2}$ distribution within the brain tissue. Adv Exp Med Biol 345:567-574.

Mallavarapu A, Mitchison T (1999) Regulated actin cytoskeleton assembly at filopodium tips controls their extension and retraction. J Cell Biol 146:1097-1106

McNally FJ (2001) CLASPing the end to the edge. Curr Biol 11: R477-R480.

Mikhailov A, Gundersen GG (1998) Relationship between microtubule dynamics and lamellipodium formation revealed by direct imaging of microtubules in cells treated with nocodazole or taxol. Cell Motil Cytoskeleton 41:325-340.

Mitchison T, Kirschner M (1984) Dynamic instability of microtubule growth. Nature 312:237-242.

O'Connor TP, Bentley D (1993) Accumulation of actin in subsets of pioneer growth cone filopodia in response to neural and epithelial guidance cues in situ. J Cell Biol 123:935-948.

O'Leary DD, Bicknese AR, De Carlos JA, Heffner CD, Koester SE, Kutka LJ, Terashima T (1990) Target selection by cortical axons: alternative mechanisms to establish axonal connections in the developing brain. Cold Spring Harb Symp Quant Biol 55:453-468.

Ren XD, Kiosses WB, Schwartz MA (1999) Regulation of the small GTP-binding protein Rho by cell adhesion and the cytoskeleton. EMBO J 18:578-585.

Rochlin MW, Wickline KM, Bridgman PC (1996) Microtubule stability decreases axon elongation but not axoplasm production. J Neurosci 16:3236-3246

Rochlin MW, Dailey ME, Bridgman PC (1999) Polymerizing microtubules activate site-directed F-actin assembly in nerve growth cones. Mol Biol Cell 10:2309-2327.

Roos J, Hummel T, Ng N, Klambt C, Davis GW (2000) Drosophila Futsch regulates synaptic microtubule organization and is necessary for synaptic growth. Neuron 26:371-382.

Sabry JH, O'Connor TP, Evans L, Toroian-Raymond A, Kirschner M, Bentley D (1991) Microtubule behavior during guidance of pioneer neuron growth cones in situ. J Cell Biol 115:381-395.

Salmon ED, Shaw SL, Waters J, Waterman-Storer CM, Maddox PS, Yeh E, Bloom K (1998) A high-resolution multimode digital microscope system. Methods Cell Biol 56:185-215.

Sanders MC, Wang YL (1991) Assembly of actin-containing cortex occurs at distal regions of growing neurites in PC12 cells. J Cell Sci 100:771-780
Schuyler SC, Pellman D (2001) Microtubule "plus-end-tracking proteins": the end is just the beginning. Cell 105:421-424.

Sider JR, Mandato CA, Weber KL, Zandy AJ, Beach D, Finst RJ, Skoble J, Bement WM (1999) Direct observation of microtubule-F-actin interaction in cell free lysates. J Cell Sci 112:1947-1956.

Spector I, Braet F, Shochet NR, Bubb MR (1999) New anti-actin drugs in the study of the organization and function of the actin cytoskeleton. Microsc Res Tech 47:18-37.

Suter DM, Forscher P (2000) Substrate-cytoskeletal coupling as a mechanism for the regulation of growth cone motility and guidance. J Neurobiol 44:97-113.

Suter DM, Errante LD, Belotserkovsky V, Forscher P (1998) The Ig superfamily cell adhesion molecule, apCAM, mediates growth cone steering by substrate-cytoskeletal coupling. J Cell Biol 141:227-240.

Szebenyi G, Callaway JL, Dent EW, Kalil K (1998) Interstitial branches develop from active regions of the axon demarcated by the primary growth cone during pausing behaviors. J Neurosci 18:7930-7940.

Szebenyi G, Dent EW, Callaway JL, Seys C, Lueth H, Kalil K (2001) FGF-2 promotes axon branching of cortical neurons by influencing morphology and behavior of the primary growth cone. J Neurosci 21:3932-3941.

Tanaka EM, Kirschner MW (1991) Microtubule behavior in the growth cones of living neurons during axon elongation. $J$ Cell Biol 115:345-363.

Tanaka E, Sabry J (1995) Making the connection: cytoskeletal rearrangements during growth cone guidance. Cell 83:171-176.

Tanaka E, Ho T, Kirschner MW (1995) The role of microtubule dynamics in growth cone motility and axonal growth. J Cell Biol 128:139-155.

Tian L, Nelson DL, Stewart DM (2000) Cdc42-interacting protein 4 mediates binding of the Wiskott-Aldrich syndrome protein to microtubules. J Biol Chem 275:7854-7861.

Tsui HT, Lankford KL, Ris H, Klein WL (1984) Novel organization of microtubules in cultured central nervous system neurons: formation of hairpin loops at ends of maturing neurites. J Neurosci 4:3002-3013.

Vasquez RJ, Howell B, Yvon AC, Wadsworth P, Cassimeris L (1997) Nanamolar concentrations of nocodazole alter microtubule dynamic instability in vivo and in vitro. Mol Biol Cell 8:973-985.

Waterman-Storer CM, Salmon ED (1998) Endoplasmic reticulum membrane tubules are distributed by microtubules in living cells using three distinct mechanisms. Curr Biol 8:798-806.

Waterman-Storer CM, Salmon E (1999) Positive feedback interactions between microtubule and actin dynamics during cell motility. Curr Opin Cell Biol 11:61-67.

Waterman-Storer CM, Desai A, Bulinski JC, Salmon ED (1998) Fluorescent speckle microscopy, a method to visualize the dynamics of protein assemblies in living cells. Curr Biol 8:1227-1230.

Waterman-Storer CM, Worthylake RA, Liu BP, Burridge K, Salmon ED (1999) Microtubule growth activates Rac1 to promote lamellipodial protrusion in fibroblasts. Nat Cell Biol 1:45-50.

Waterman-Storer C, Duey DY, Weber KL, Keech J, Cheney RE, Salmon ED, Bement WM (2000) Microtubules remodel actomyosin networks in Xenopus egg extracts via two mechanisms of F-actin transport. J Cell Biol 150:361-376.

Welnhofer EA, Zhao L, Cohan CS (1997) Actin dynamics and organization during growth cone morphogenesis in Helisoma neurons. Cell Motil Cytoskeleton 37:54-71.

Williamson T, Gordon-Weeks PR, Schachner M, Taylor J (1996) Microtubule reorganization is obligatory for growth cone turning. Proc Natl Acad Sci USA 93:15221-15226.

Wills Z, Marr L, Zinn K, Goodman CS, Van Vactor D (1999) Profilin and the Abl tyrosine kinase are required for motor axon outgrowth in the Drosophila embryo. Neuron 22:291-299.

Yvon AC, Wadsworth P, Jordan AM (1999) Taxol suppresses dynamics of individual microtubules in living human tumor cells. Mol Biol Cell 10:947-959. 